TRANSACTIONS OF THE

AMERICAN MATHEMATICAL SOCIETY

Volume 351, Number 3, March 1999, Pages 1109-1121

S 0002-9947(99)02071-1

\title{
A WEAKLY CHAINABLE TREE-LIKE CONTINUUM WITHOUT THE FIXED POINT PROPERTY
}

\author{
PIOTR MINC
}

\begin{abstract}
An example of a fixed points free map is constructed on a tree-like, weakly chainable continuum.
\end{abstract}

\section{INTRODUCTION}

It is still unknown whether every nonseparating plane continuum has the fixed point property. This old question was asked by Sternbach in 1935, The Scottish Book [21, Problem 107], but it clearly motivated even earlier papers by W. Scherrer (1926 [30]), W. L. Ayres (1930 [1]), G. Nöbeling (1932 [26]) and K. Borsuk (1932 [7]). Since then many more partial solutions, both in positive and negative directions, have been published. In particular, O. Hamilton [14] proved that every chainable continuum has the fixed point property. (A continuum is chainable if it is the inverse limit of an inverse sequence of arcs.) Note that every chainable continuum can be embedded in the plane (see [5]). H. Bell [2], K. Sieklucki [31] and S. Iliadis [15] proved that every nonseparating plane continuum with no indecomposable continuum in its boundary has the fixed point property. C. Hagopian [12] proved that each nonseparating arcwise connected plane continuum does not contain an indecomposable continuum in its boundary, and therefore has the fixed point property. The author proved that every nonseparating weakly chainable plane continuum also has the fixed point property [22]. (A continuum is weakly chainable if it is a continuous image of a chainable continuum; see [18], [10] and [25].)

Nonseparating plane continua are cell-like. In 1935, K. Borsuk [8] constructed an example of a cell-like continuum in $\mathbf{R}^{3}$ without the fixed point property. Twodimensional examples were then constructed by S. Kinoshita (contractible [16]), R. Knill [17] and R. H. Bing [6]. Bing [5, p. 653] [6] asked whether a tree-like continuum without the fixed point property could be constructed. (A continuum is tree-like if it is the inverse limit of a sequence of trees.) D. P. Bellamy [3] answered this question affirmatively by presenting in 1978 his spectacular example.

The Bellamy continuum and other tree-like continua without the fixed point property constructed subsequently (see [27], [28], [29], [23] and [24]) are not (at least appear not to be) weakly chainable. In 1983 Bellamy asked whether every weakly chainable tree-like continuum has the fixed point property [19, Problem 36]; see also [4]. A positive answer to this question seemed likely in view of the

Received by the editors May 20, 1996 and, in revised form, March 6, 1997.

1991 Mathematics Subject Classification. Primary 54F15; Secondary 54H25.

Key words and phrases. Tree-like continuum, weakly chainable, fixed point property.

This research was supported in part by NSF grant \# DMS-9505108.

The author is grateful to Charles L. Hagopian for suggesting several valuable improvements. 


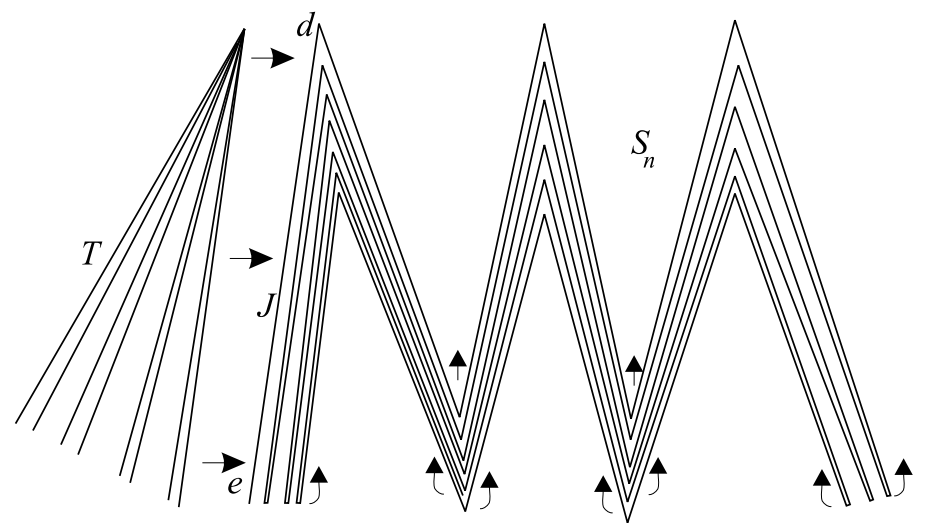

FIGURE 1

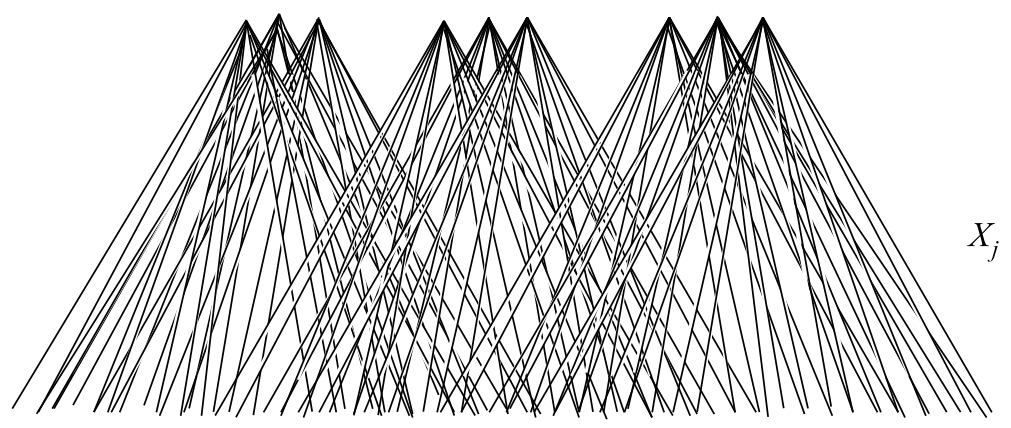

FiguRE 2

theorem in the plane and a classic result by K. Borsuk who, in 1954, proved the fixed point theorem for arcwise-connected tree-like continua [9]. The last result was even further extended by R. Mańka [20] to hereditarily decomposable tree-like continua. Very recently, C. Hagopian [13] proved that every map of a tree-like continuum that sends each arc-component into itself has a fixed point. It turns out, however, that Bellamy's construction can be modified to answer his question in the negative. More precisely, we prove the following theorem:

Theorem 1.1. For each positive integer $j$, there is a weakly chainable tree-like continuum $X_{j}$ and a map $\bar{f}_{j}: X_{j} \rightarrow X_{j}$ with no fixed points and no periodic points with periods less than or equal to $j$.

The idea of the modification is very simple. To construct the Bellamy continuum, which is denoted here by $B_{j}$, start with $S_{n}$, a copy of the " $n$-fold horseshoe" contained in the $x y$-plane (see Figure 1 ). Let $J$ denote the "basic arc" containing the endpoint $e$ of $S_{n}$. Let $d$ denote the other endpoint of $J$ and let $P$ be the plane containing $J$ and perpendicular to the $x y$-plane. Remove the arc $J$ from $S_{n}$ and replace it by a cone $T$ over a certain 0 -dimensional set in such a way that $T$ is contained in $P$ and the vertex of $T$ replaces $d$. Simultaneously, reshape the remainder of $S_{n}$ by keeping the upper endpoint of each "basic arc" fixed on the $x y$-plane and raising the other endpoint to the level of a certain endpoint of $T$. In 
the resulting continuum $B_{j}$, each "basic arc" of $S_{n} \backslash J$ is almost parallel to one of the $\operatorname{arcs}$ forming $T$. To get weak chainability, we replace each "basic arc" by a copy of $T$ (see Figure 2).

The map $\bar{f}_{j}$ is not a homeomorphism. Applying to $\bar{f}_{j}$ a technique by J. B. Fugate and L. B. Mohler [11], we get a homeomorphism of a tree-like continuum $\widetilde{B}_{j}$ which may not be (and probably is not) weakly chainable. For this reason, $X_{j}$ and $\bar{f}_{j}$ cannot be used in a construction similar to the one in [24] to get a weakly chainable tree-like continuum $X_{j}$ admitting a map without periodic points of all periods.

\section{Construction of $X_{j}$}

In this paper we will keep the notation from [23]. In particular, $j, n, v, g_{i}, S_{n}$, $g, E, D, e, d, H_{0}, w, r, B_{j}$ and $f_{j}$ will denote the same objects as in [23]. We will recall most of the definitions as they are needed.

Recall that $v$ folds uniformly the real line onto the interval $[0,1]$ such that $v(i)=$ 0 for each even integer $i$ and $v(i)=1$ for each odd $i$. For each positive integer $k$, $g_{k}$ is the map stretching the interval $[0,1] k$ times and then folding it uniformly back onto itself such that $g_{k}(s)=v(k s)$ for each $s \in[0,1]$. Recall also that $j$ is a positive integer and $n=2\left(4^{1}-1\right)\left(4^{2}-1\right) \ldots\left(4^{j}-1\right)$.

For each $i=0,1, \ldots$, let $I_{i}$ denote the interval $[0,1]$. If $0 \leq m \leq i$, let $p_{m i}: I_{i} \rightarrow$ $I_{m}$ denote the map $\left(g_{n}\right)^{i-m}$. Let $S_{n}$ be the inverse limit of the system $\left\{I_{i}, p_{m i}\right\}$ and let $p_{i}$ denote the projection of $S_{n}$ onto $I_{i}$.

If $m$ and $i$ are integers such that $0 \leq m \leq i$, then $p_{m i}$ restricted to the closure of any component of $\left(p_{m i}\right)^{-1}((0,1))$ is a homeomorphism onto $I_{m}$. It follows that $p_{m}$ restricted to the closure of any component of $\left(p_{m}\right)^{-1}((0,1))$ is a homeomorphism onto $I_{m}$.

For each $s \in[0,1]$, let $A_{i}(s)$ denote the set of $\left(p_{0 i}\right)^{-1}(s)$. Let $A_{i}, E_{i}$ and $D_{i}$ denote $A_{i}\left(\frac{1}{2}\right), A_{i}(0)$ and $A_{i}(1)$, respectively.

For each $s \in I_{i} \backslash\left(E_{i} \cup D_{i}\right)$, let $J_{i}(s)$ denote the closure of the component of $s$ in $I_{i} \backslash\left(E_{i} \cup D_{i}\right)$. Let $e_{i}(s) \in E_{i}$ and $d_{i}(s) \in D_{i}$ denote the endpoints of $J_{i}(s)$.

Observe that if $m$ and $i$ are integers such that $0 \leq m \leq i$, and $a \in A_{i}$, then $p_{m i}(a) \in A_{m}$,

$$
\begin{aligned}
& J_{m}\left(p_{m i}(a)\right)=p_{m i}\left(J_{i}(a)\right) \\
& e_{m}\left(p_{m i}(a)\right)=p_{m i}\left(e_{i}(a)\right)
\end{aligned}
$$

and

$$
d_{m}\left(p_{m i}(a)\right)=p_{m i}\left(d_{i}(a)\right) .
$$

Since $n$ is even, for each positive integer $i$ and each point $a \in A_{i}$, there is exactly one point $\delta_{i}(a) \in A_{i} \backslash\{a\}$ such that $d_{i}(a)=d_{i}\left(\delta_{i}(a)\right)$. Additionally, set $d_{0}\left(\frac{1}{2}\right)=\frac{1}{2}$.

Observe that if $m$ and $i$ are integers such that $0 \leq m \leq i$, and $a \in A_{i}$, then

$$
p_{m i}\left(\delta_{i}(a)\right)=\delta_{m}\left(p_{m i}(a)\right)
$$

If $a \in A_{i}$ and $\frac{3}{2 n^{i}} \leq a \leq 1-\frac{3}{2 n^{i}}$, then there is exactly one point $\epsilon_{i}(a) \in A_{i} \backslash\{a\}$ such that $e_{i}(a)=e_{i}\left(\epsilon_{i}(a)\right)$. Additionally, set $\epsilon_{i}(a)=a$, if either $a=\frac{1}{2 n^{i}}$ or $a=1-\frac{1}{2 n^{i}}$. 
Notice that if $m$ and $i$ are integers such that $0 \leq m \leq i$, and $a \in A_{i}$, then

$$
p_{m i}\left(\epsilon_{i}(a)\right)=\epsilon_{m}\left(p_{m i}(a)\right) .
$$

For each $s \in[0,1]$, let $A(s)$ denote the set of $p_{0}^{-1}(s)$. By $A, E$ and $D$ we denote the sets $A\left(\frac{1}{2}\right), A(0)$ and $A(1)$. Notice that $E$ and $D$ coincide with those defined in $[23]$.

If $x \in S_{n} \backslash(E \cup D)$, let $J(x)$ denote the closure of the component of $x$ in $S_{n} \backslash(E \cup D)$. If $0 \leq m \leq i$, then $p_{m i}$ restricted to $J_{i}\left(p_{i}(x)\right)$ is a homeomorphism onto $J_{m}\left(p_{m}(x)\right)$. It follows that $p_{0}$ restricted to $J(x)$ is a homeomorphism onto $[0,1]$, and therefore $J(x)$ is an arc with one endpoint $e(x) \in E$ and the other $d(x) \in D$. Let $e$ denote the endpoint of $S_{n}$, that is the point such that $p_{i}(e)=0$ for each $i=0,1, \ldots$ Let $a_{0}$ denote the point of $A$ such that $e\left(a_{0}\right)=e$ and let $d$ denote $d\left(a_{0}\right)$. Notice that $e$ and $d$ coincide with those defined in [23].

Observe that, for each point $a \in A$, there is exactly one point $\delta(a) \in A \backslash\{a\}$ such that $d(a)=d(\delta(a))$. Similarly, for each point $a \in A \backslash\left\{a_{0}\right\}$, there is exactly one point $\epsilon(a) \in A \backslash\{a\}$ such that $e(a)=e(\epsilon(a))$. Additionally, set $\epsilon\left(a_{0}\right)=a_{0}$. Clearly, $\delta(\delta(a))=a$ and $\epsilon(\epsilon(a))=a$. Observe also that $\delta(a)$ and $\epsilon(a)$ depend continuously on the choice of $a$.

Since $p_{i}(A)=A_{i}, p_{i}(E)=E_{i}, p_{i}(D)=D_{i}$ and $p_{i}$ restricted to the closure of any component of $\left(p_{i}\right)^{-1}((0,1))$ is a homeomorphism onto $I_{i}$, we have that

$$
p_{i}(\delta(a))=\delta_{i}\left(p_{i}(a)\right)
$$

and

$$
p_{i}(\epsilon(a))=\epsilon_{i}\left(p_{i}(a)\right) .
$$

Let $\bar{S}_{j}$ be $[0,1] \times A$ with the following identifications:

$(1, a)$ is identified with $(1, \delta(a))$ for each $a \in A$ and

$(0, a)$ is identified with $(0, \epsilon(a))$ for each $a \in A \backslash\left\{a_{0}\right\}$.

Let $h: \bar{S}_{j} \rightarrow S_{j}$ be the map such that $h(s, a) \in J(a)$ and $p_{0}(h(s, a))=s$ for each $s \in[0,1]$ and each $a \in A$. Clearly, $h$ is a homeomorphism of $\bar{S}_{j}$ onto $S_{j}$.

Since $g_{2} \circ g_{n}=g_{n} \circ g_{2}$, the maps $g_{2}: I_{i} \rightarrow I_{i}$ induce a map $g: S_{n} \rightarrow S_{n}$ (see [23]). Recall that $H_{0}$, defined in [23], is a 0-dimensional compactum, $r: H_{0} \rightarrow H_{0}$ and $w: E \backslash\{e\} \rightarrow H_{0}$ are continuous maps such that $r \circ w=w \circ g$.

Let $T_{0}$ be $H_{0} \times[0,1]$ with $H_{0} \times\{1\}$ identified to a point. We will denote by $\alpha$ the natural projection of $H_{0} \times[0,1]$ onto $T_{0}$. Let $t_{0}$ denote the point $\alpha\left(H_{0} \times\{1\}\right)$. Let $\pi_{0}$ denote the projection of $T_{0}$ onto the interval $[0,1]$. For each point $a \in A \backslash\left\{a_{0}\right\}$ and each $s \in[0,1]$, let $w_{s}(a)$ denote the point $\alpha(w(e(a)), s)$.

Since $e(\epsilon(a))=e(a)$, we have that $w_{s}(a)=w_{s}(\epsilon(a))$.

Let $X_{j}$ be $T_{0} \times A$ with the following identifications:

$\left(t_{0}, a\right)$ is identified with $\left(t_{0}, \delta(a)\right)$ for each $a \in A$ and

$\left(w_{0}(a), a\right)$ is identified with $\left(w_{0}(\epsilon(a)), \epsilon(a)\right)$ for each $a \in A \backslash\left\{a_{0}\right\}$.

We will denote by $\kappa$ the natural projection of $T_{0} \times A$ onto $X_{j}$. Let $T=\kappa\left(T_{0} \times\left\{a_{0}\right\}\right)$ and $t_{1}=\kappa\left(t_{0}, a_{0}\right)$.

Let $\bar{\pi}: X_{j} \rightarrow \bar{S}_{n}$ be defined in the following way: if $x=\kappa(t, a)$ for $(t, a) \in$ $T_{0} \times A$, then let $\bar{\pi}(x)$ be the point in $\bar{S}_{n}$ representing $\left(\pi_{0}(t), a\right)$. Notice that $\bar{\pi}$ is a continuous map. Let $\pi=h \circ \bar{\pi}$.

We will define $\chi:\left(S_{n} \backslash J\left(a_{0}\right)\right) \cup\{d\} \rightarrow\left(X_{j} \backslash T\right) \cup\left\{t_{1}\right\}$ by the following: if $x \in J(a)$ for $a \in A \backslash\left\{a_{0}\right\}$ and $s=p_{0}(x)$, then $\chi(x)=\kappa\left(w_{s}(a), a\right)$. Observe 
that $\chi$ is a continuous map of $\left(S_{n} \backslash J\left(a_{0}\right)\right) \cup\{d\}$ and $\pi \circ \chi$ is the identity map on $\left(S_{n} \backslash J\right) \cup\{d\}$. Let $B_{j}$ denote $\chi\left(\left(S_{n} \backslash J\right) \cup\{d\}\right) \cup T$. Observe that so defined $B_{j}$ coincides with the one defined in [23].

\section{Construction of $\bar{f}_{j}$}

For an arbitrary point $a$ of $A=A\left(\frac{1}{2}\right)$, let $a_{-}$and $a_{+}$denote the points $A\left(\frac{1}{4}\right) \cap$ $J(a)$ and $A\left(\frac{3}{4}\right) \cap J(a)$, respectively. Note that $a_{-}$and $a_{+}$depend continuously on $a$. Since $g: S_{n} \rightarrow S_{n}$ is the map induced by $g_{2}: I_{i} \rightarrow I_{i}$, we have that $g(a) \in D$, $g\left(a_{-}\right) \in A$ and $g\left(a_{+}\right) \in A$.

The following proposition can be easily verified and its proof is omitted here.

Proposition 3.1. Suppose $b \in J(a)$ and $s=p_{0}(b)$. Then $p_{0}(g(b))=g_{2}(s)$. Moreover, $g(b) \in J\left(g\left(a_{-}\right)\right)$if $0 \leq s \leq \frac{1}{2}$, and $g(b) \in J\left(g\left(a_{+}\right)\right)$if $\frac{1}{2} \leq s \leq 1$.

Since $g(J(a))=J\left(g\left(a_{-}\right)\right) \cup J\left(g\left(a_{+}\right)\right)$and $g(a) \in D$,

$$
\delta\left(g\left(a_{-}\right)\right)=g\left(a_{+}\right) .
$$

Since $g(e(a)) \in E$ is an endpoint of $J\left(g\left(a_{-}\right)\right)$,

$$
e\left(g\left(a_{-}\right)\right)=g(e(a)) .
$$

Substituting $\epsilon(a)$ instead of $a$ in (3.3) we get

$$
e\left(g\left([\epsilon(a)]_{-}\right)\right)=g(e(\epsilon(a))) .
$$

Since $J(a)=J\left(a_{-}\right)$intersects $J(\epsilon(a))=J\left([\epsilon(a)]_{-}\right)$at $e(a)$, the $\operatorname{arcs} J\left(g\left(a_{-}\right)\right)$ and $J\left(g\left([\epsilon(a)]_{-}\right)\right)$intersect at $g(e(a)) \in E$. It follows that

$$
\epsilon\left(g\left([\epsilon(a)]_{-}\right)\right)=g\left(a_{-}\right) .
$$

Since $J(a)=J\left(a_{+}\right)$intersects $J(\delta(a))=J\left([\delta(a)]_{+}\right)$at $d(a)$, the $\operatorname{arcs} J\left(g\left(a_{+}\right)\right)$ and $J\left(g\left([\delta(a)]_{+}\right)\right)$intersect at $g(d(a)) \in E$. It follows that

$$
\epsilon\left(g\left([\delta(a)]_{+}\right)\right)=g\left(a_{+}\right) .
$$

We will define a function $\bar{f}_{j}: X_{j} \rightarrow X_{j}$ in the following way. For an arbitrary $x \in X_{j}$, let $a \in A, z \in H_{0}$ and $s \in[0,1]$ be such that $x=\kappa(\alpha(z, s), a)$. Let

$$
\bar{f}_{j}(x)= \begin{cases}\kappa\left(\alpha(r(z), 2 s), g\left(a_{-}\right)\right), & \text {for } 0 \leq s \leq \frac{1}{2}, \\ \kappa\left(w_{2-2 s}\left(g\left(a_{+}\right)\right), g\left(a_{+}\right)\right), & \text {for } \frac{1}{2} \leq s \leq 1 .\end{cases}
$$

Proposition 3.7. Every nonendpoint of $X_{j}$ is eventually moved to $B_{j}$ by some iteration of $\bar{f}_{j}$.

Proof of 3.7. Every nonendpoint $x$ of $X_{j}$ is either contained in $B_{j}$ or it is of the form $x=\kappa(\alpha(z, s), a)$, where $s>0$. Let $k$ be an integer such that $s 2^{k} \geq 1$. Observe that $\bar{f}_{j}^{k}(x) \in B_{j}$.

We will show that

Proposition 3.8. $\bar{f}_{j}$ is continuous.

Proof of 3.8. Notice that the choice $s$ is unique. Since every function appearing in the definition of $\bar{f}_{j}$ is continuous, it is enough to show that the function is well defined. For this purpose we need to show 
3.8.1. If $s=\frac{1}{2}$, then the first line of the definition of $\bar{f}_{j}$ yields the same result as the second.

3.8.2. The definition of $\bar{f}_{j}$ does not depend on the choice of a and $z$ describing the same $x$.

Proof of 3.8.1. Since $s=\frac{1}{2}$, we have that $\alpha(r(z), 2 s)=\alpha(r(z), 1)=t_{0}$ and $w_{2-2 s}\left(g\left(a_{+}\right)\right)=w_{1}\left(g\left(a_{+}\right)\right)=t_{0}$. According to the first line of the definition $\bar{f}_{j}(x)=\kappa\left(\alpha(r(z), 2 s), g\left(a_{-}\right)\right)=\kappa\left(t_{0}, g\left(a_{-}\right)\right)=\kappa\left(t_{0}, \delta\left(g\left(a_{-}\right)\right)\right)$. By (3.2), the last point is the same as $\kappa\left(t_{0}, g\left(a_{+}\right)\right)=\kappa\left(w_{2-2 s}\left(g\left(a_{+}\right)\right), g\left(a_{+}\right)\right)$which is $\bar{f}_{j}(x)$ evaluated by the second line of the definition of $\bar{f}_{j}$.

Proof of 3.8.2. Suppose that $a^{\prime} \in A$ and $z^{\prime} \in H_{0}$ are such that $\kappa\left(\alpha\left(z^{\prime}, s\right), a^{\prime}\right)=$ $x=\kappa(\alpha(z, s), a)$. Let $y$ and $y^{\prime}$ denote the values resulting from applying the definition of $\bar{f}_{j}$ to the triples $z, s, a$ and $z^{\prime}, s, a^{\prime}$, respectively. We will show that $y^{\prime}=y$. It is enough to consider the following two cases:

Case 1. $s=0, a^{\prime}=\epsilon(a)$ and $z=z^{\prime}=w(e(a))=w(e(\epsilon(a)))$.

Case 2. $s=1$ and $a^{\prime}=\delta(a)$.

Proof of 3.8.2 assuming Case 1. Observe that

$$
y^{\prime}=\kappa\left(\alpha\left(r\left(z^{\prime}\right), 0\right), g\left(\left[a^{\prime}\right]_{-}\right)\right)=\kappa\left(\alpha(r(w(e(\epsilon(a)))), 0), g\left([\epsilon(a)]_{-}\right)\right) .
$$

Since $r \circ w=w \circ g$, we have the result that

$$
r(w(e(\epsilon(a))))=w(g(e(\epsilon(a)))) .
$$

By (3.4),

$$
w(g(e(\epsilon(a))))=w\left(e\left(g\left([\epsilon(a)]_{-}\right)\right)\right)
$$

and consequently $\alpha(r(w(e(\epsilon(a)))), 0)=w_{0}\left(g\left([\epsilon(a)]_{-}\right)\right)$. Thus,

$$
y^{\prime}=\kappa\left(w_{0}\left(g\left([\epsilon(a)]_{-}\right)\right), g\left([\epsilon(a)]_{-}\right)\right) .
$$

By the definition of $X_{j}$ we get that

$$
y^{\prime}=\kappa\left(w_{0}\left(\epsilon\left(g\left([\epsilon(a)]_{-}\right)\right)\right), \epsilon\left(g\left([\epsilon(a)]_{-}\right)\right)\right) .
$$

By (3.5),

$$
y^{\prime}=\kappa\left(w_{0}\left(g\left(a_{-}\right)\right), g\left(a_{-}\right)\right)=\kappa\left(\alpha\left(w\left(e\left(g\left(a_{-}\right)\right)\right), 0\right), g\left(a_{-}\right)\right) .
$$

Applying (3.3) to the last expression we get that

$$
y^{\prime}=\kappa\left(\alpha(w(g(e(a))), 0), g\left(a_{-}\right)\right) .
$$

Since $w \circ g=r \circ w$,

$$
y^{\prime}=\kappa\left(\alpha(r(w(e(a))), 0), g\left(a_{-}\right)\right)=y
$$

Proof of 3.8.2 assuming Case 2. It follows from the definition of $X_{j}$ that

$$
y^{\prime}=\kappa\left(w_{0}\left(g\left(\left[a^{\prime}\right]_{+}\right)\right), g\left(\left[a^{\prime}\right]_{+}\right)\right)=\kappa\left(w_{0}\left(\epsilon\left(g\left(\left[a^{\prime}\right]_{+}\right)\right)\right), \epsilon\left(g\left(\left[a^{\prime}\right]_{+}\right)\right)\right) .
$$

Now, apply (3.6) to get that $\epsilon\left(g\left(\left[a^{\prime}\right]_{+}\right)\right)=\epsilon\left(g\left([\delta(a)]_{+}\right)\right)=g\left(a_{+}\right)$. Thus

$$
y^{\prime}=\kappa\left(w_{0}\left(\epsilon\left(g\left(\left[a^{\prime}\right]_{+}\right)\right)\right), \epsilon\left(g\left(\left[a^{\prime}\right]_{+}\right)\right)\right)=\kappa\left(w_{0}\left(g\left(a_{+}\right)\right), g\left(a_{+}\right)\right)=y .
$$


Remark 3.9. $\bar{f}_{j}$ restricted to $B_{j}$ is equal to $f_{j}$ defined in [23].

Proposition 3.10. The diagram

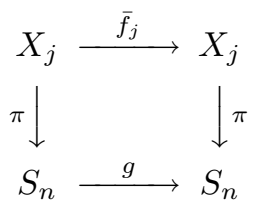

commutes.

Proof of 3.10. Let $x$ be an arbitrary point in $X_{j}$. Let $a \in A, z \in H_{0}$ and $s \in[0,1]$ be such that $x=\kappa(\alpha(z, s), a) . \pi(x)$ is the only point of $J(a)$ such that $p_{0}(\pi(x))=s$. By Proposition 3.1, $p_{0}(g(\pi(x)))=g_{2}(s)$. Recall that $g_{2}(s)=2 s$ if $0 \leq s \leq \frac{1}{2}$, and $g_{2}(s)=2-2 s$ if $\frac{1}{2} \leq s \leq 1$.

If $0 \leq s \leq \frac{1}{2}$, then $g(\pi(x)) \in J\left(g\left(a_{-}\right)\right)$. In this case,

$$
\bar{f}_{j}(x)=\kappa\left(\alpha(r(z), 2 s), g\left(a_{-}\right)\right) .
$$

It follows that $\pi\left(\bar{f}_{j}(x)\right)$ also belongs to $J\left(g\left(a_{-}\right)\right)$and $p_{0}\left(\pi\left(\bar{f}_{j}(x)\right)\right)=2 s=g_{2}(s)$. So, $\pi\left(\bar{f}_{j}(x)\right)=g(\pi(x))$ if $0 \leq s \leq \frac{1}{2}$.

If $\frac{1}{2} \leq s \leq 1$, then $g(\pi(x)) \in J\left(g\left(a_{+}\right)\right)$. In this case,

$$
\bar{f}_{j}(x)=\kappa\left(w_{2-2 s}\left(g\left(a_{+}\right)\right), g\left(a_{+}\right)\right)=\kappa\left(\alpha\left(w\left(e\left(g\left(a_{+}\right)\right)\right), 2-2 s\right), g\left(a_{+}\right)\right) .
$$

It follows that $\pi\left(\bar{f}_{j}(x)\right)$ belongs to $J\left(g\left(a_{+}\right)\right)$and $p_{0}\left(\pi\left(\bar{f}_{j}(x)\right)\right)=2-2 s=g_{2}(s)$. So, $\pi\left(\bar{f}_{j}(x)\right)=g(\pi(x))$.

Proposition 3.11. $\bar{f}_{j}$ has no fixed points and no periodic points with periods less than or equal to $j$. Moreover, all periodic points of $\bar{f}_{j}$ are contained in $B_{j}$.

Proof of 3.11. The first part of the proposition follows from Proposition 3.10 and Propositions 2.6 and 2.10 in [23]. The second part of Proposition 3.11 is a simple consequence of $3.7,3.9$ above and 2.10 in [23].

\section{InVERSE Limit DESCRIPTION OF $X_{j}$}

There exists a sequence $\mathcal{K}_{0}, \mathcal{K}_{1} \ldots$ such that for each $i=0,1, \ldots$ the following conditions are satisfied:

$\mathcal{K}_{i}$ is a collection of mutually disjoint nonempty, closed subsets of $H_{0}$ filling up $H_{0}$,

$\mathcal{K}_{i+1}$ refines $\mathcal{K}_{i}$ and

$\lim _{i \rightarrow \infty} \operatorname{mesh}\left(\mathcal{K}_{i}\right)=0$.

For $0 \leq m \leq i$ and $u \in \mathcal{K}_{i}$, let $\varphi_{m i}(u)$ be the element of $\mathcal{K}_{m}$ containing $u$. Notice that $H_{0}$ may be naturally considered as the inverse limit of the system $\left\{\mathcal{K}_{i}, \varphi_{m i}\right\}$. Let $\varphi_{i}$ be the projection of $H_{0}$ onto $\mathcal{K}_{i}$. Observe that if $x \in H_{0}$ then $\varphi_{i}(x)$ is the unique element of $\mathcal{K}_{i}$ containing $x$.

We will construct a strictly increasing sequence of integers $\mu(0), \mu(1), \mu(2), \ldots$ such that for each positive integer $i$ and each $b \in E_{\mu(i)} \backslash\left(p_{\mu(i-1) \mu(i)}\right)^{-1}(0)$, the set $w\left(\left(p_{\mu(i)}\right)^{-1}(b)\right)$ is contained in a single element $w^{i}(b)$ of $\mathcal{K}_{\mu(i-1)}$.

Let $\mu(0)=1$. Suppose $\mu(i-1)=m$ has been constructed. We will construct $\mu(i)$. Since the set $E \backslash\left(p_{m}\right)^{-1}(0)$ is compact, there is a positive number $\eta$ such that if a set $S \subset E \backslash\left(p_{m}\right)^{-1}(0)$ has the diameter less than $\eta$ then $w(S)$ is contained in 
a single element of $\mathcal{K}_{m}$. There is an integer $k>m$ such that diam $\left(\left(p_{k}\right)^{-1}(b)\right)<\eta$ for each $b \in I_{k}$. If $b \in E_{k} \backslash\left(p_{m k}\right)^{-1}(0)$, then $\left(p_{k}\right)^{-1}(b) \subset E \backslash\left(p_{m}\right)^{-1}(0)$, and consequently $w\left(\left(p_{k}\right)^{-1}(b)\right)$ is contained in a single element of $\mathcal{K}_{m}$. So, we may set $\mu(i)=k$.

In order to simplify the notation we will write $p_{m i}^{*}, p_{i}^{*}, \epsilon_{i}^{*}, \delta_{i}^{*}$ and $e_{i}^{*}$ instead of $p_{\mu(m) \mu(i)}, p_{\mu(i)}, \epsilon_{\mu(i)}, \delta_{\mu(i)}$ and $e_{\mu(i)}$, respectively.

Let $G_{i}$ denote the set $\left\{a \in A_{\mu(i)} \mid e_{i}^{*}(a) \in\left(p_{i-1 i}^{*}\right)^{-1}(0)\right\}$. Since

$$
e_{i}^{*}(a)=e_{i}^{*}\left(\epsilon_{i}^{*}(a)\right)
$$

we have that

Proposition 4.1. $a \in G_{i}$ if and only if $\epsilon_{i}^{*}(a) \in G_{i}$.

Proposition 4.2. Suppose $m$ and $i$ are integers such that $1 \leq m \leq i$. Suppose also $a$ is an element of $A_{\mu(i)} \backslash G_{i}$ such that $p_{m i}^{*}(a) \notin G_{m}$. Then

$$
\varphi_{\mu(m-1) \mu(i-1)}\left(w^{i}\left(e_{i}^{*}(a)\right)\right)=w^{m}\left(e_{m}^{*}\left(p_{m i}^{*}(a)\right)\right) .
$$

Proof of 4.2. Since $p_{m}^{*}=p_{m i}^{*} \circ p_{i}^{*}$, we get from (2.2) that

$$
\left(p_{i}^{*}\right)^{-1}\left(e_{i}^{*}(a)\right) \subset\left(p_{m}^{*}\right)^{-1}\left(e_{m}^{*}\left(p_{m i}^{*}(a)\right)\right) .
$$

Thus $w^{m}\left(e_{m}^{*}\left(p_{m i}^{*}(a)\right)\right)$, the element of $\mathcal{K}_{\mu(m-1)}$ containing $\left(p_{m}^{*}\right)^{-1}\left(e_{m}^{*}\left(p_{m i}^{*}(a)\right)\right)$, must contain $w^{i}\left(e_{i}^{*}(a)\right)$, the element of $\mathcal{K}_{\mu(i-1)}$ containing $\left(p_{i}^{*}\right)^{-1}\left(e_{i}^{*}(a)\right)$.

Proposition 4.3. Suppose $a \in A$ and $i=0,1, \ldots$ are such that $p_{i}^{*}(a) \notin G_{i}$. Then

$$
\varphi_{\mu(i-1)}(w(e(a)))=w^{i}\left(e_{i}^{*}\left(p_{i}^{*}(a)\right)\right) .
$$

Proof of 4.3. Since $p_{i}^{*}(e(a))=e_{i}^{*}\left(p_{i}^{*}(a)\right)$, we have that

$$
e(a) \in\left(p_{i}^{*}\right)^{-1}\left(e_{i}^{*}\left(p_{i}^{*}(a)\right)\right) .
$$

Thus $w^{i}\left(e_{i}^{*}\left(p_{i}^{*}(a)\right)\right)$ is the element of $\mathcal{K}_{\mu(i-1)}$ containing $w(e(a))$.

For each positive integer $i$, let $T_{0}^{i}$ be $\mathcal{K}_{\mu(i-1)} \times[0,1]$ with $\mathcal{K}_{\mu(i-1)} \times\{1\}$ identified to a point. We will denote by $\alpha_{i}$ the natural projection of $\mathcal{K}_{\mu(i-1)} \times[0,1]$ onto $T_{0}^{i}$. Let $t_{0}^{i}$ denote the point $\alpha_{i}\left(\mathcal{K}_{\mu(i-1)} \times\{1\}\right)$.

For $1 \leq m \leq i$ and $z_{i} \in T_{0}^{i}$ we will define $\psi_{m i}\left(z_{i}\right) \in T_{0}^{m}$ by choosing $u_{i} \in \mathcal{K}_{\mu(i-1)}$ and $s \in[0,1]$ such that $\alpha_{i}\left(u_{i}, s\right)=z_{i}$ and setting

$$
\psi_{m i}\left(z_{i}\right)=\alpha_{m}\left(\varphi_{\mu(m-1) \mu(i-1)}\left(u_{i}\right), s\right) .
$$

Note that $\psi_{m i}$ is a well-defined map of $T_{0}^{i}$ onto $T_{0}^{m}$. Observe also that $T_{0}$ may be naturally considered as the inverse limit of the system $\left\{\mathcal{T}_{0}^{i}, \psi_{m i}\right\}$. We will denote by $\psi_{i}$ the projection of $T_{0}$ onto $T_{0}^{i}$.

Suppose $a \in A_{\mu(i)} \backslash G_{i}$. Clearly, $e_{i}^{*}(a) \in E_{\mu(i)} \backslash\left(p_{i-1 i}^{*}\right)^{-1}(0)$ and $w^{i}\left(e_{i}^{*}(a)\right)$ is defined. Let $w_{0}^{i}(a)$ denote $\alpha_{i}\left(w^{i}\left(e_{i}^{*}(a)\right), 0\right)$. Since $e_{i}^{*}(a)=e_{i}^{*}\left(\epsilon_{i}^{*}(a)\right)$, it is true that $w_{0}^{i}(a)=w_{0}^{i}\left(\epsilon_{i}^{*}(a)\right)$.

Proposition 4.4. Suppose $m$ and $i$ are integers such that $1 \leq m \leq i$. Let $a \in A_{\mu(i)}$ be such that $p_{m i}^{*}(a) \notin G_{m}$. Then

$$
\psi_{m i}\left(w_{0}^{i}(a)\right)=w_{0}^{m}\left(p_{m i}^{*}(a)\right) .
$$


Proof of 4.4. Since $p_{m i}^{*}(a) \notin G_{m}$, it follows that $a \notin G_{i}$ and consequently both $w_{0}^{i}(a)$ and $w_{0}^{m}\left(p_{m i}^{*}(a)\right)$ are well-defined. Observe that

$$
\psi_{m i}\left(w_{0}^{i}(a)\right)=\psi_{m i}\left(\alpha_{i}\left(w^{i}\left(e_{i}^{*}(a)\right), 0\right)\right)=\alpha_{m}\left(\varphi_{\mu(m-1) \mu(i-1)}\left(w^{i}\left(e_{i}^{*}(a)\right)\right), 0\right) .
$$

By 4.2, the last expression is equal to $\alpha_{m}\left(w^{m}\left(e_{m}^{*}\left(p_{m i}^{*}(a)\right)\right), 0\right)=w_{0}^{m}\left(p_{m i}^{*}(a)\right)$.

Proposition 4.5. Suppose $a \in A$ and $i$ is a positive integer such that $p_{i}^{*}(a) \notin G_{i}$. Then

$$
\psi_{i}\left(w_{0}(a)\right)=w_{0}^{i}\left(p_{i}^{*}(a)\right) .
$$

Proof of 4.5. $\psi_{i}\left(w_{0}(a)\right)=\psi_{i}(\alpha(w(e(a)), 0))=\alpha_{i}\left(\varphi_{\mu(i-1)}(w(e(a))), 0\right)$. By 4.3, the last expression is equal to

$$
\alpha_{i}\left(w^{i}\left(e_{i}^{*}\left(p_{i}^{*}(a)\right)\right), 0\right)=w_{0}^{i}\left(p_{i}^{*}(a)\right) .
$$

For each positive integer $i$, let $X_{j}^{i}$ be $T_{0}^{i} \times A_{\mu(i)}$ with the following identifications: $\left(t_{0}^{i}, a\right)$ is identified with $\left(t_{0}^{i}, \delta_{i}^{*}(a)\right)$ for each $a \in A_{\mu(i)}$, $\left(w_{0}^{i}(a), a\right)$ is identified with $\left(w_{0}^{i}\left(\epsilon_{i}^{*}(a)\right), \epsilon_{i}^{*}(a)\right)$ for each $a \in A_{\mu(i)} \backslash G_{i}$ and

$(u, a)$ is identified with $\left(u, \epsilon_{i}^{*}(a)\right)$ for each $u \in T_{0}^{i}$ and each $a \in G_{i}$.

We will denote by $\kappa_{i}$ the natural projection of $T_{0}^{i} \times A_{\mu(i)}$ onto $X_{j}^{i}$.

The following proposition follows from (2.4).

Proposition 4.6. Suppose $m$ and $i$ are integers such that $1 \leq m \leq i$. Let $a \in A_{i}$. Then

$$
\kappa_{m}\left(\psi_{m i}\left(t_{0}^{i}\right), p_{m i}^{*}(a)\right)=\kappa_{m}\left(\psi_{m i}\left(t_{0}^{i}\right), p_{m i}^{*}\left(\delta_{m}^{*}(a)\right)\right) .
$$

Proposition 4.7. Suppose $m$ and $i$ are integers such that $1 \leq m \leq i$. Let $a \in$ $A_{\mu(i)} \backslash G_{i}$. Then

$$
\kappa_{m}\left(\psi_{m i}\left(w_{0}^{i}(a)\right), p_{m i}^{*}(a)\right)=\kappa_{m}\left(\psi_{m i}\left(w_{0}^{i}\left(\epsilon_{i}^{*}(a)\right)\right), p_{m i}^{*}\left(\epsilon_{i}^{*}(a)\right)\right) .
$$

Proof of 4.7. Suppose $p_{m i}^{*}(a) \in G_{m}$. Then the point $\psi_{m i}\left(w_{0}^{i}(a), p_{m i}^{*}(a)\right)$ is identified with $\psi_{m i}\left(w_{0}^{i}(a), \epsilon_{m}^{*}\left(p_{m i}^{*}(a)\right)\right)$ by $\kappa_{m}$. By $(2.5), p_{m i}^{*}\left(\epsilon_{i}^{*}(a)\right)=\epsilon_{m}^{*}\left(p_{m i}^{*}(a)\right)$. Since $w_{0}^{i}\left(\epsilon_{i}^{*}(a)\right)=w_{0}^{i}(a)$, the proposition is in this case trivial. Thus, we may assume that $p_{m i}^{*}(a) \notin G_{m}$.

By Proposition 4.4,

$$
\kappa_{m}\left(\psi_{m i}\left(w_{0}^{i}(a)\right), p_{m i}^{*}(a)\right)=\kappa_{m}\left(w_{0}^{m}\left(p_{m i}^{*}(a)\right), p_{m i}^{*}(a)\right) .
$$

Applying Proposition 4.1 and $(2.5)$ we get that $p_{m i}^{*}\left(\epsilon_{i}^{*}(a)\right) \notin G_{m}$. Again by Proposition 4.4 ,

$$
\kappa_{m}\left(\psi_{m i}\left(w_{0}^{i}\left(\epsilon_{i}^{*}(a)\right)\right), p_{m i}^{*}\left(\epsilon_{i}^{*}(a)\right)\right)=\kappa_{m}\left(w_{0}^{m}\left(p_{m i}^{*}\left(\epsilon_{i}^{*}(a)\right)\right), p_{m i}^{*}\left(\epsilon_{i}^{*}(a)\right)\right) .
$$

Applying 2.5, we get that the last expression is equal to

$$
\kappa_{m}\left(w_{0}^{m}\left(\epsilon_{m}^{*}\left(p_{m i}^{*}(a)\right)\right), \epsilon_{m}^{*}\left(p_{m i}^{*}(a)\right)\right) .
$$

Since $\left(w_{0}^{m}\left(p_{m i}^{*}(a)\right), p_{m i}^{*}(a)\right)$ and $\left(w_{0}^{m}\left(\epsilon_{m}^{*}\left(p_{m i}^{*}(a)\right)\right), \epsilon_{m}^{*}\left(p_{m i}^{*}(a)\right)\right)$ are identified by $\kappa_{m}$, the proposition is true. 
For any two integers $m$ and $i$ such that $1 \leq m \leq i$, we will define $\sigma_{m i}: X_{j}^{i} \rightarrow X_{j}^{m}$ in the following way. For an arbitrary $x \in X_{j}^{i}$, let $a \in A_{\mu(i)}$ and $z \in T_{0}^{i}$ be such that $x=\kappa_{i}(z, a)$. Let $\sigma_{m i}(x)=\kappa_{m}\left(\psi_{m i}(z), p_{m i}^{*}(a)\right)$. It follows from 4.6 and 4.7 that $\sigma_{m i}$ is a well-defined continuous map.

Proposition 4.8. The continuum $X_{j}$ is homeomorphic to the inverse limit of the system $\left\{X_{j}^{i}, \sigma_{m i}\right\}$.

Proof of Proposition 4.8. For each positive integer $i$, we will define $\sigma_{i}: X_{j} \rightarrow X_{j}^{i}$ in the following way. For an arbitrary $x \in X_{j}$, take $z \in T_{0}$ and $a \in A$ such that $x=\kappa(z, a)$. Let $\sigma_{i}(x)=\kappa_{i}\left(\psi_{i}(z), p_{i}^{*}(a)\right)$. An easy proof that $\sigma_{i}$ is a welldefined map, not depending on the choice of $z$ and $a$, follows from (2.6), (2.7) and Proposition 4.3, and will be omitted here.

Claim. Suppose $x=\kappa(z, a)$ and $x^{\prime}=\kappa\left(z^{\prime}, a^{\prime}\right)$ are two different points of $X_{j}$. Then there is a positive integer $k$ such that $\sigma_{k}(x) \neq \sigma_{k}\left(x^{\prime}\right)$.

Proof of Claim. Since $A$ and $T_{0}$ are the inverse limits of the systems $\left\{A_{\mu(i)}, p_{m i}^{*}\right\}$ and $\left\{T_{0}^{i}, \psi_{m i}\right\}$, respectively, there is a positive integer $k$ satisfying the following conditions:

1. if $a^{\prime} \neq a$, then $p_{k}^{*}\left(a^{\prime}\right) \neq p_{k}^{*}(a)$,

2. if $a^{\prime} \neq \delta(a)$, then $p_{k}^{*}\left(a^{\prime}\right) \neq p_{k}^{*}(\delta(a))$,

3. if $a^{\prime} \neq \epsilon(a)$, then $p_{k}^{*}\left(a^{\prime}\right) \neq p_{k}^{*}(\epsilon(a))$,

4. if $a \neq a_{0}$, then $p_{k}^{*}(a) \notin G_{k}$,

5. if $a^{\prime} \neq a_{0}$, then $p_{k}^{*}\left(a^{\prime}\right) \notin G_{k}$,

6. if $z^{\prime} \neq z$, then $\psi_{k}\left(z^{\prime}\right) \neq \psi_{k}(z)$.

Now, it is easy to prove using (2.6) and (2.7) that the points $\left(\psi_{k}(z), p_{k}^{*}(a)\right)$ and $\left(\psi_{k}\left(z^{\prime}\right), p_{k}^{*}\left(a^{\prime}\right)\right)$ are not among the pairs of points identified by $\kappa_{k}$.

Observe that for each integer $m$ and $i$ such that $1 \leq m \leq i$, we have that $\sigma_{m}=\sigma_{m i} \circ \sigma_{i}$. For each $x \in X_{j}$, let $\sigma(x)=\left(\sigma_{1}(x), \sigma_{2}(x), \ldots\right)$. Clearly, $\sigma$ is a continuous map of $X_{j}$ into the inverse limit of the system $\left\{X_{j}^{i}, \sigma_{m i}\right\}$. Since $\sigma_{i}$ maps $X_{j}$ onto $X_{j}^{i}$, the map $\sigma$ is surjective. It follows from the claim that $\sigma$ is a homeomorphism.

Let $\mathcal{C}_{i}$ denote the collection of sets $\kappa_{i}\left(T_{0}^{i} \times\{a\}\right)$ where $a \in A_{\mu(i)}$. Clearly, if $\kappa_{i}\left(T_{0}^{i} \times\{a\}\right)=\kappa_{i}\left(T_{0}^{i} \times\left\{a^{\prime}\right\}\right)$, then both $a$ and $a^{\prime}$ belong to $G_{i}$ and $a^{\prime}=\epsilon_{i}^{*}(a)$. Let $\lambda(i)$ be the number of distinct elements of $\mathcal{C}_{i}$. Arrange elements of $\mathcal{C}_{i}$ into a sequence $C_{i}^{1}, C_{i}^{2}, \ldots, C_{i}^{\lambda(i)}$ in such a way that if $k$ and $k^{\prime}$ are integers and $a, a^{\prime}$ are two elements of $A_{\mu(i)}$ such that $1 \leq k<k^{\prime} \leq \lambda(i), C_{i}^{k}=\kappa_{i}\left(T_{0}^{i} \times\{a\}\right)$ and $C_{i}^{k^{\prime}}=\kappa_{i}\left(T_{0}^{i} \times\left\{a^{\prime}\right\}\right)$, then $a<a^{\prime}$.

Observe that for each integer $i \geq 2, \sigma_{i-1 i}$ maps each element of $\mathcal{C}_{i}$ onto an element of $\mathcal{C}_{i-1}$. For $k=1, \ldots, \lambda(i)$, let $\tau(k, i)$ denote the integer such that $1 \leq \tau(k, i) \leq \lambda(i-1)$ and $\sigma_{i-1 i}\left(C_{i}^{k}\right)=C_{i-1}^{\tau(k, i)}$. Clearly, $|\tau(k, i)-\tau(k-1, i)| \leq 1$ for each $k=2, \ldots, \lambda(i)$. Observe also that, for each $k=1, \ldots, \lambda(i)-1$, the intersection $C_{i}^{k} \cap C_{i}^{k+1}$ consists of one point. We will denote this point by $c_{i}^{k}$.

The following proposition is a simple consequence of the construction.

Proposition 4.9. Suppose $L^{\prime}$ and $L$ are closed intervals contained in the real line. Let $b_{0}$ and $b_{1}$ be the endpoints of $L$. Suppose $\beta^{\prime}$ is a map of $L^{\prime}$ onto $C_{i-1}^{\tau(k, i)}$. Let $c_{0}, c_{1} \in C_{i}^{k}$ and $b_{0}^{\prime}, b_{1}^{\prime} \in L^{\prime}$ be such that $\sigma_{i-1 i}\left(c_{0}\right)=\beta^{\prime}\left(b_{0}^{\prime}\right)$ and $\sigma_{i-1 i}\left(c_{1}\right)=\beta^{\prime}\left(b_{1}^{\prime}\right)$ 
Then there is a map $\gamma_{k}: L \rightarrow L^{\prime}$ and there is a map $\beta_{k}$ of $L$ onto $C_{i}^{k}$ such that $\gamma_{k}\left(b_{0}\right)=b_{0}^{\prime}, \gamma_{k}\left(b_{1}\right)=b_{1}^{\prime}, \beta_{k}\left(b_{0}\right)=c_{0}, \beta_{k}\left(b_{1}\right)=c_{1}$ and $\beta^{\prime} \circ \gamma_{k}=\sigma_{i-1 i} \circ \beta_{k}$.

Proof of 4.9. Let $a_{i}^{k}$ denote an element of $A_{\mu(i)}$ such that $C_{i}^{k}=\kappa_{i}\left(T_{0}^{i} \times\left\{a_{i}^{k}\right\}\right)$. Let $\mathcal{Z}$ be the collection of components of $C_{i}^{k} \backslash\left\{\kappa_{i}\left(t_{0}^{i}, a_{i}^{k}\right)\right\}$. Let $\left\{L_{z}\right\}_{z \in \mathcal{Z}}$ be a collection of mutually disjoint subintervals contained in the interior of $L$. Let $\gamma_{k}: L \rightarrow L^{\prime}$ be such that $\gamma_{k}\left(b_{0}\right)=b_{0}^{\prime}, \gamma_{k}\left(b_{1}\right)=b_{1}^{\prime}$ and $\gamma_{k}\left(L_{z}\right)=L^{\prime}$ for each $z \in \mathcal{Z}$.

Since $\sigma_{i-1 i}$ restricted to each $z \in \mathcal{Z}$ is a homeomorphism onto $\sigma_{i-1 i}(z)$, the map $\beta^{\prime} \circ \gamma_{k}$ can be lifted through $\sigma_{i-1 i}$ to map $\beta_{k}: L \rightarrow C_{i}^{k}$ such that $\beta_{k}\left(b_{0}\right)=c_{0}$, $\beta_{k}\left(b_{1}\right)=c_{1}$ and $\beta^{\prime} \circ \gamma_{k}=\sigma_{i-1 i} \circ \beta_{k}$. The map $\beta_{k}$ can be made surjective by guaranteeing that $z \subset \beta_{k}\left(L_{z}\right)$ for each $z \in \mathcal{Z}$.

Proposition 4.10. Let $s_{m}$ denote $\frac{m}{\lambda(i-1)}$ for $m=0,1, \ldots, \lambda(i-1)$ and let $u_{k}$ denote $\frac{k}{\lambda(i)}$ for $k=0,1, \ldots, \lambda(i)$. Suppose $\omega_{i-1}$ is a map of the interval $[0,1]$ onto $X_{j}^{i-1}$ such that $\omega_{i-1}\left(\left[s_{m-1}, s_{m}\right]\right)=C_{i-1}^{m}$ for $m=1, \ldots, \lambda(i-1)$. Then there are maps $\omega_{i}:[0,1] \rightarrow X_{j}^{i}$ and $\xi_{i}:[0,1] \rightarrow[0,1]$ such that $\sigma_{i-1 i} \circ \omega_{i}=\omega_{i-1} \circ \xi_{i}$ and $\omega_{i}\left(\left[u_{k-1}, u_{k}\right]\right)=C_{i}^{k}$ for $k=1, \ldots, \lambda(i)$.

Proof of 4.10. Observe that $\omega_{i-1}\left(s_{m}\right)=c_{i-1}^{m}$ for $m=1, \ldots, \lambda(i-1)-1$.

For each $k=1, \ldots, \lambda(i)$, we will define two maps: a map $\gamma_{k}:\left[u_{k-1}, u_{k}\right] \rightarrow\left[s_{\tau(k, i)-1}, s_{\tau(k, i)}\right]$ and a map $\beta_{k}$ of $\left[u_{k-1}, u_{k}\right]$ onto $C_{i}^{k}$ such that the diagram

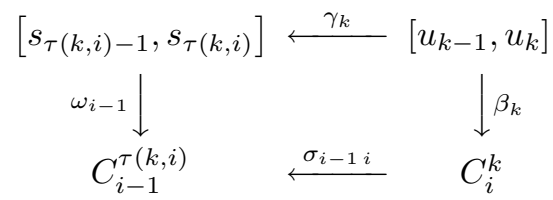

is commutative,

(4.10.2) $\beta_{k}\left(u_{k}\right)=c_{i}^{k}$ for $k=1, \ldots, \lambda(i)-1$,

(4.10.3) $\beta_{k}\left(u_{k-1}\right)=c_{i}^{k-1}$ for $k=2, \ldots, \lambda(i)$,

(4.10.4) $\gamma_{k}\left(u_{k-1}\right)=\gamma_{k-1}\left(u_{k-1}\right)$ for $k=2, \ldots, \lambda(i)$,

(4.10.5) if $\tau(k, i)<\tau(k+1, i)$, then $\gamma_{k}\left(u_{k}\right)=s_{\tau(k, i)}$ for $k=1, \ldots, \lambda(i)-1$ and

(4.10.6) if $\tau(k, i)>\tau(k+1, i)$, then $\gamma_{k}\left(u_{k}\right)=s_{\tau(k, i)-1}$ for $k=1, \ldots, \lambda(i)-1$.

In order to construct $\beta_{1}$ and $\gamma_{1}$, set $k=1, L^{\prime}=\left[s_{0}, s_{1}\right], b_{0}=u_{0}, b_{1}=u_{1}$, $\beta^{\prime}=\omega_{i-1}\left\lceil L^{\prime}, b_{0}^{\prime}=s_{0}, b_{1}^{\prime}=s_{1}\right.$ and $c_{1}=c_{i}^{1}$. Additionally, let $c_{0}$ be a point of $C_{i}^{1}$ such that $\sigma_{i-1} i\left(c_{0}\right)=\omega_{i-1}\left(s_{0}\right)$. Now, apply Proposition 4.9 to get $\beta_{1}$ and $\gamma_{1}$.

Suppose $\beta_{1}, \ldots, \beta_{k-1}$ and $\gamma_{1}, \ldots, \gamma_{k-1}$ satisfying (4.10.1)-(4.10.6) have been constructed. We will observe that

$$
\gamma_{k-1}\left(u_{k-1}\right) \in\left[s_{\tau(k, i)-1}, s_{\tau(k, i)}\right] .
$$

One of the following three cases is true: either

$$
\begin{aligned}
& 1 \tau(k-1, i)=\tau(k, i)-1 \text { or } \\
& 2 \tau(k-1, i)=\tau(k, i) \text { or } \\
& 3 \tau(k-1, i)=\tau(k, i)+1 .
\end{aligned}
$$

In the first case, by (4.10.5) for $k-1, \gamma_{k-1}\left(u_{k-1}\right)=s_{\tau(k-1, i)}=s_{\tau(k, i)-1} \cdot(4.10 .7)$ is obvious in the second case. Finally, in the third case, by (4.10.6) for $k-1$, $\gamma_{k-1}\left(u_{k-1}\right)=s_{\tau(k-1, i)-1}=s_{\tau(k, i)}$. 
We will now construct $\beta_{k}$ and $\gamma_{k}$ by applying Proposition 4.9 again. Set $L^{\prime}=$ $\left[s_{\tau(k, i)-1}, s_{\tau(k, i)}\right], b_{0}=u_{k-1}, b_{1}=u_{k}$ and $\beta^{\prime}=\omega_{i-1} \uparrow L^{\prime}$. Let $b_{0}^{\prime}=\gamma_{k-1}\left(u_{k-1}\right)$ and $c_{0}=c_{i}^{k-1}$. By (4.10.7), $b_{0}^{\prime} \in L^{\prime}$. It follows from (4.10.1) and (4.10.2) for $k-1$ that $\sigma_{i-1 i}\left(c_{0}\right)=\beta^{\prime}\left(b_{0}^{\prime}\right)$.

Let $c_{1}=c_{i}^{k}$ if $k<\lambda(i)$ and let $c_{1}$ be any point of $C_{i}^{k}$ if $k=\lambda(i)$. Before we apply Proposition 4.9 we must define $b_{1}^{\prime}$ in such a way that $\sigma_{i-1 i}\left(c_{1}\right)=\beta^{\prime}\left(b_{1}^{\prime}\right)$. We will consider the following three cases:

Case 1: either $k=\lambda(i)$ or $\tau(k, i)=\tau(k+1, i)$,

Case 2: $\tau(k, i)=\tau(k+1, i)-1$ and

Case 3: $\tau(k, i)=\tau(k+1, i)+1$.

In the first case $b_{1}^{\prime}$ may be defined as any element of $L^{\prime}$ such that $\omega_{i-1}\left(b_{1}^{\prime}\right)=$ $\sigma_{i-1 i}\left(c_{1}\right)$. In the second case, set $b_{1}^{\prime}=s_{\tau(k, i)}$ and notice that $\omega_{i-1}\left(b_{1}^{\prime}\right)=c_{i-1}^{\tau(k, i)}=$ $\sigma_{i-1 i}\left(c_{i}^{k}\right)$. Finally, in the third case, set $b_{1}^{\prime}=s_{\tau(k, i)-1}$ and notice that $\omega_{i-1}\left(b_{1}^{\prime}\right)=$ $c_{i-1}^{\tau(k, i)-1}=\sigma_{i-1 i}\left(c_{i}^{k}\right)$.

Now, observe that Proposition 4.9 yields $\beta_{k}$ and $\gamma_{k}$ satisfying (4.10.1)-(4.10.6).

Let the functions $\omega_{i}$ and $\xi_{i}$ be defined by $\omega_{i}(z)=\beta_{k}(z)$ and $\xi_{i}(z)=\gamma_{k}(z)$ for $z \in\left[u_{k-1}, u_{k}\right]$, where $k=1, \ldots, \lambda(i)$. It follows from (4.10.1)-(4.10.4) that $\omega_{i}$ and $\xi_{i}$ are well-defined maps satisfying the proposition.

Proposition 4.11. $X_{j}$ is weakly chainable.

Proof of 4.11. Let $\omega_{0}$ be a map of $[0,1]$ onto $X_{j}^{0}$. Use Proposition 4.10 repeatedly to get two sequences of maps $\xi_{1}, \xi_{2}, \ldots$ and $\omega_{1}, \omega_{2}, \ldots$ such that $\xi_{i}:[0,1] \rightarrow[0,1]$, $\omega_{i}$ maps $[0,1]$ onto $X_{j}^{i}$ for each positive integer $i$ and the diagram

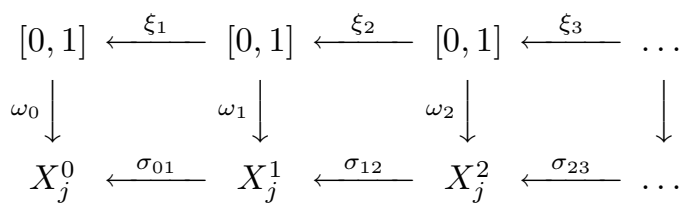

is commutative. The diagram induces a map from the inverse limit of the sequence $\left\{[0,1], \xi_{i}\right\}$ onto $X_{j}$ which is the inverse limit of $\left\{X_{j}^{i}, \sigma_{m i}\right\}$.

The proof of Theorem 1.1 follows from 3.8, 3.11, 4.8 and 4.11.

\section{REFERENCES}

1. W. L. Ayres, Some generalizations of the Scherrer fixed-point theorem, Fund. Math. 16 (1930), 332-336.

2. H. Bell, On fixed point properties of plane continua, Trans. Amer. Math. Soc. 128 (1967), 539-548. MR 35:4888

3. D.P. Bellamy, A tree-like continuum without the fixed-point property, Houston J.Math. 6 (1980), 1-13. MR 81h:54039

4. - The fixed point property in dimension one, in Continua with the Houston problem book (H. Cook et al., eds.), Marcel Dekker, New York, 1995, pp. 27-35. MR 96a:54056

5. R.H. Bing, Snake-like continua, Duke Math. J. 18 (1951), 653-663. MR 13:265a

6. 38:5201

7. K. Borsuk, Einige Sätze über stetige Streckenbilder, Fund. Math. 18 (1932), 198-213.

8. __ Sur un continu acyclique qui se laisse transformer topologiquement en lui même sans points invariants, Fund. Math. 24 (1935), 51-58.

9. __ A theorem on fixed points, Bull. Acad. Sci. Polon. 2 (1954), 17-20. MR 16:275h 
10. L. Fearnley, Characterizations of continuous images of pseudoarc, Trans. Amer. Math. Soc. 111 (1964), 380-399. MR 29:596

11. J. B. Fugate and L. A. Mohler, A note on fixed points in tree-like continua, Topology Proc. 2 (1977), 457-460. MR 80k:54062

12. C. L. Hagopian, A fixed point theorem for plane continua, Bull. Amer. Math. Soc. 77 (1971), 351-354. MR 42:8469

13.

14. O. H. Hamilton, A fixed point theorem for pseudoarcs and certain other metric continua, Proc. Amer. Math. Soc 2 (1951), 173-174. MR 12:627f

15. S. Iliadis, Positions of continua in a plane and fixed points, Vestn. Moskov. Univ. 25 (1970), 66-70. MR 44:4726

16. S. Kinoshita, On some contractible continua without fixed point property, Fund. Math. 40 (1953), 96-98. MR 15:642b

17. R. Knill, Cones, products and fixed points, Fund. Math. 60 (1967), 35-46. MR 35:2270

18. A. Lelek, On weakly chainable continua, Fund Math. 51 (1962), 271-282. MR 26:742

19. W. Lewis, Continuum theory problems, Topology Proc. 8 (1983), 361-394. MR 86a:54038

20. R. Mańka, Association and fixed points, Fund. Math. 91 (1976), 105-121. MR 54:1183

21. R. D. Mauldin (ed.), The Scottish Book: Mathematics from the Scottish Café, Birkhauser, Boston, 1981. MR 84m:00015

22. P. Minc, A fixed point theorem for weakly chainable plane continua, Trans. Amer. Math. Soc. 317 (1990), 303-312. MR 90d:54067

23. _ A tree-like continuum admitting fixed point free maps with arbitrarily small trajectories, Topology and its Appl. 46 (1992), 99-106. MR 94a:54108

24. Soc. 348 (1996), 1487-1519. MR 96h:54029

25. J. Mioduszewski, A functional conception of snake-like continua, Fund. Math. 51 (1962), 179-189. MR 26: 1859

26. G. Nöbeling, Eine Fixpunkteigenschaft der BaumKurven, Ergebnisse eines mathematischen Kolloquium 2 (1932), 19.

27. L.G. Oversteegen and J.T. Rogers, Jr., Tree-like continua as limits of cyclic graphs, Topology Proc. 4 (1979), 507-515. MR 82c:54032

28. __ An inverse limit description of an atriodic tree-like continuum and an induced map without a fixed point, Houston J.Math. 6 (1980), 549-564. MR 82j:54075

29. _ Fixed-point-free maps on tree-like continua, Topology and its Appl. 13 (1982), 85-95. MR 83b:54044

30. W. Scherrer, Über ungeschlossene stetige Kurven, Math. Zeitschr. 24 (1926), 125-130.

31. K. Sieklucki, On a class of plane acyclic continua with the fixed point property, Fund. Math. 63 (1968), 257-278. MR 39:2139

Department of Mathematics, Auburn University, Auburn, Alabama 36849

E-mail address: mincpio@mail. auburn.edu 